\title{
La competencia genérica de reconocimiento y aprecio por la diversidad humana: Evaluación en la formación inicial de docentes ${ }^{1}$
}

\author{
Generic Competency for the Acknowledgement and Appreciation for Human Diversity: \\ Evaluation of Professors' Initial Training ${ }^{2}$
}

\section{Competência genérica para o reconhecimento e apreço da diversidade humana: Avaliação na formação inicial de professores ${ }^{3}$}

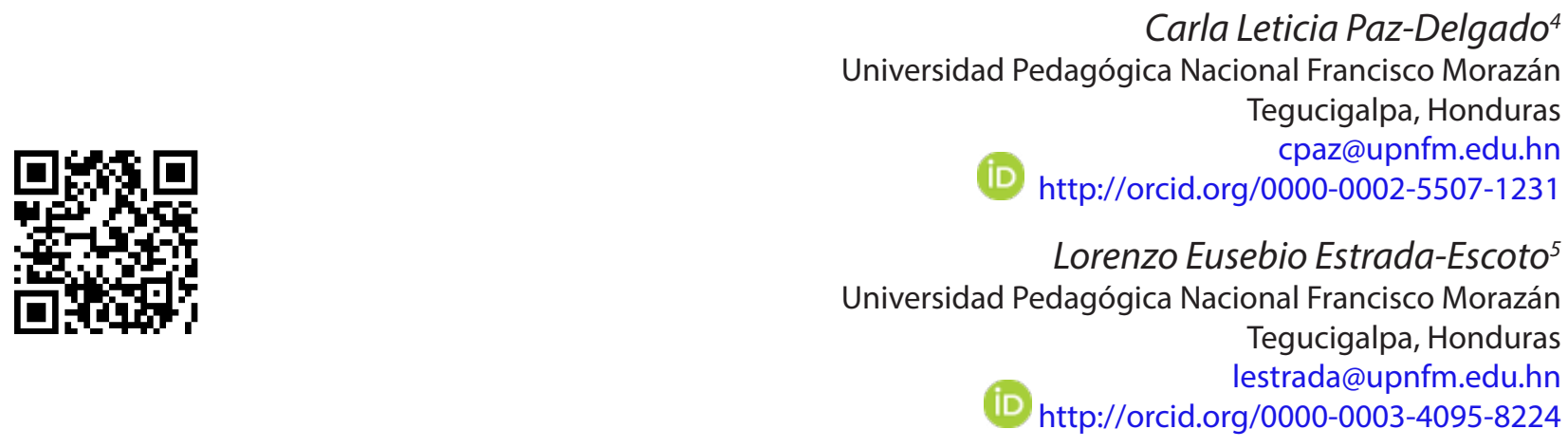

Recibido 29 de abril de 2016 • Corregido 8 de agosto de 2017 • Aceptado 27 de agosto de 2017 Received 29 de abril de 2016 • Revised 8 de agosto de 2017 • Accepted 27 de agosto de 2017 Recebido 29 de abril de 2016 • Revisado 8 de agosto de 2017 • Aprovado 27 de agosto de 2017

\footnotetext{
${ }^{1}$ Este proyecto de investigación ha sido patrocinado por el Fondo de Apoyo a la Investigación de la UPNFM.

${ }^{2}$ This research Project has been sponsored by the Research Funds-UPNFM.

${ }^{3}$ Este projeto de pesquisa foi patrocinada pelo Fondo de Apoyo a la Investigación da UPNFM.

${ }^{4}$ Es licenciada en Educación Especial y magister en Investigación Educativa por la Universidad Pedagógica Nacional Francisco Morazán (UPNFM). Posee un doctorado en Investigación Educativa: Enseñanza y Aprendizaje por la Universidad de Alicante, España y es especialista en Investigación en el Fenómeno de las Drogas por la Universidad de San Pablo, Brasil. Se ha desempeñado como asistente de la Dirección de Desarrollo Curricular de la UPNFM y como docente de la Carrera de Educación Especial por 15 años. Actualmente es coordinadora de investigación del Instituto de Investigación y Evaluación Educativas y Sociales (INIEES) de la UPNFM.

${ }^{5}$ Es licenciado en Educación Comercial por la Universidad Pedagógica Nacional Francisco Morazán (UPNFM) y Máster en Administración y Finanzas por la Universidad Nacional Autónoma de Honduras. Se ha desempeñado como docente en el Centro de Investigación e Innovación Educativas (CIIE), docente en el Departamento de Educación Comercial. Actualmente es jefe de la Sección Académica de Educación Comercial en el Sistema de Educación a Distancia de la UPNFM.
} 
doi: http://dx.doi.org/10.15359/ree.21-3.19

URL: http://www.una.ac.cr/educare

CORREO: educare@una.cr

Resumen: Es innegable que la profesión docente, en la actualidad, se lleva a cabo en entornos educativos, sociales y culturales altamente diversos. El reconocimiento de este hecho ha propiciado que la formación inicial y permanente del profesorado se oriente hacia la educación en la diversidad y para esta. En este sentido, la Universidad Pedagógica Nacional Francisco Morazán (UPNFM) incluyó, en la reciente reforma de sus planes de estudio, una competencia genérica o transversal, orientada a contribuir a la formación del personal docente que aprecie y perciba la diversidad como un valor enriquecedor del proceso educativo. La finalidad de esta investigación era identificar el nivel de desarrollo de esta competencia, para proponer una estrategia que permita visibilizar como transversales los saberes propios de una educación inclusiva. El proyecto ha sido desarrollado desde la perspectiva de investigación para la mejora y la toma de decisiones, con un enfoque mixto, utilizando el análisis documental, la entrevista en profundidad y el cuestionario como técnicas de recolección de datos. En el estudio participaron 225 estudiantes de práctica profesional II y 8 docentes de la formación de fundamento. Los resultados indican que, en la UPNFM, a nivel de diseño curricular se ha logrado un avance en la formación del personal docente para la atención a la diversidad, incorporando al plan de estudios una competencia vinculada con la educación inclusiva; sin embargo, el personal docente y el estudiantado señalaron carecer de habilidades para la aplicación de estrategias didácticas pertinentes con la atención a las diferencias humanas. Ello implica fortalecer el proceso de implementación curricular a través de la formación permanente de docentes universitarios.

Palabras claves: Competencias genéricas; formación inicial de docentes; diversidad; inclusión.

Abstract: Currently, teaching takes place in highly diverse social, educational and cultural environments. Understanding and accepting this fact has allowed that pre-service and in-service teaching training be taught as education for diversity. It is in this sense that the Universidad Pedagógica Nacional Francisco Morazán (UPNFM, Pedagogical University Francisco Morazán) has, in a recent curricular reform, included a generic and transverse competency to contribute to the training of the University faculty, so that they can appreciate and understand diversity as an enriching asset of the educational process. The main objective of this research is to identify the level of development of this competency to propose a strategy to visualize inclusive education as transverse knowledge. This project was developed from the perspective of research for improvement and decision making, using a mixed methodology and collecting data through the analysis of documents, in-depth interviews, and a questionnaire. The participants in the study were 225 students, who were in their professional internship II, and eight professors from the General Ed courses.

The results showed that UPNFM, in its curricular offer, has achieved great improvements as for the training of their faculty members regarding attention to diversity by incorporating in its curriculum a competency linked to an inclusive education. However, both members of the faculty and students pointed out that they did not have the skills to apply teaching strategies suitable to deal with diversity. Therefore, it is necessary to strengthen the process of curricular implementation through ongoing training of university professors.

Keywords: Generic competencies; initial training of teachers; diversity; inclusion. 


\begin{abstract}
Resumo: É inegável que atualmente o trabalho docente é realizado em ambientes educacionais, sociais e culturais diversos. O reconhecimento desta realidade ajudou a que a formação inicial e permanente de professores seja orientada para a educação na diversidade. A este respeito, na recente reforma dos planos de estudo da Universidade Pedagógica Nacional Francisco Morazán (UPNFM) foram incluídos no seu currículo uma competência genérica ou transversal, orientada a ajudar na formação dos professores para que apreciem e percebam a diversidade como um valor enriquecedor do processo formativo. O objetivo desta pesquisa foi identificar o nível de desenvolvimento desta competência, com a finalidade de propor uma estratégia que permita visibilizar como transversal os saberes da educação inclusiva. O projeto foi desenvolvido com perspectiva de uma pesquisa para melhorar a tomada de decisão, com uma abordagem mista, fazendo uma análise documental, entrevistas em profundidade e questionário como técnicas de coleta de dados. Na pesquisa participaram 225 estudantes de Prática Profissional II e 8 professores da área de Fundamentação. Os resultados indicam que na UPNFM, em quanto ao currículo, percebem-se progressos na formação de professores para a atenção à diversidade, incorporando ao plano de estudos uma competência vinculada à educação inclusiva; no entanto, os professores e os estudantes manifestaram que ainda faltam melhorar as habilidades para a implementação de estratégias didáticas relevantes que brinda atenção as diferenças humanas. Isso exige o fortalecimento do processo de implementação do currículo através da formação contínua dos professores universitários.
\end{abstract}

Palavras chaves: competências genéricas; formação inicial de professores; diversidade; inclusão.

\title{
Introducción
}

Ante el hecho real de la diversidad humana, se reconoce que las diferencias intelectuales, étnicas, de lengua, de costumbres, etc. existieron y existen y no pueden dejar de ser consideradas sino en el marco de la democracia (De Valle y Vega, 2006). Sin embargo, Luque y Delgado (2002) explican que tanta diversidad produce más complejidad de la que es cómodo manejar. Así que hemos aprendido a través del tiempo a simplificarla en categorías sociales y, en la medida en que existen desigualdades en la realidad social, aprendemos a clasificary valorar a las personas en función de los rasgos que, de acuerdo con las ideologías que vamos asimilando, definen su condición.

La diferencia se ha usado como una forma de segregación, en lugar de celebración. El cuidado que se debe tener al hablar de las diferencias es básico, pues siempre se terminan generando categorías que en lugar de ser útiles siempre crean situaciones de segregación (Thomas y Loxley, 2007). Por estas razones, se habla de asumir una filosofía de la diferencia, en la cual el concepto hace referencia a una condición general de la humanidad como valor positivo en la sociedad, como un elemento enriquecedor para los grupos humanos (Artiles, 1998).

En educación, por mucho tiempo, se consideró que aprender era una experiencia individual, por lo tanto, no fue posible el desarrollo de una escuela democrática en la cual se reconocieran y valoraran las diferencias. A pesar del reconocimiento de la diversidad, la teoría y práctica escolar se siguen desarrollando desde un enfoque tradicional, que busca crear nuevas etiquetas y patrones homogeneizadores (García, 2005). 
doi: http://dx.doi.org/10.15359/ree.21-3.19

URL: http://www.una.ac.cr/educare

CORREO: educare@una.cr

Sin embargo, los movimientos de renovación pedagógica y el establecimiento de políticas a nivel global, como la Declaración de Salamanca (Unesco, 1994), la Declaración de Educación para Todos (Unesco, 1990), han gestado la atención a la diversidad desde el marco de la educación inclusiva. Este hecho ha ocasionado el replanteamiento de la formación del profesorado y, por ello, hoy en día, son numerosos los esfuerzos por crear modelos, planes y programas de formación docente para la atención a la diversidad desde contextos inclusivos (CERI 2010; Ball y Tyson, 2011).

No fue sino hasta la década de los noventa, coincidiendo con el movimiento de la inclusión educativa, cuando se comenzó a plantear con seriedad que los planes de formación debían incorporar módulos o componentes para la atención a la diversidad (Blanton, Griffin, Winn, y Pugach, 1996). La reforma a los programas de formación inicial docente ha sido ampliamente recomendada, como un medio para mejorar la preparación de las futuras generaciones de docentes en atención a la diversidad (Van Laarhoven, Munk, Lynch, Bosma, y Rouse, 2007).

Frente a este panorama, la Universidad Pedagógica Nacional Francisco Morazán (UPNFM), consciente de su compromiso en la formación de cuadros docentes que promuevan experiencias educativas de calidad, efectuó una reforma a los planes de estudio y, en el año 2009, inició su implementación. En la actualidad, la formación en las carreras de pregrado de la UPNFM se desarrolla desde el enfoque curricular basado en competencias académico- profesionales. En el perfil del graduado o graduada se ha incluido una competencia genérica orientada a desarrollar la capacidad de convivir en paz, promover el respeto a la diversidad, multiculturalidad y los derechos humanos. Este es un paso firme de la UPNFM hacia la formación del personal docente, capaz de reconocer, apreciar y atender las diferencias humanas, desde el marco de la educación inclusiva.

A pesar de este importante avance se hace necesario crear las condiciones didácticas, para asegurar que esta competencia sea desarrollada por el profesorado universitario. Un estudio anterior reveló que el profesorado en la UPNFM reconoce la necesidad de formarse en el ámbito de la educación para la diversidad (Paz, 2006).

Precisamente ese escenario ha motivado el desarrollo de esta investigación, cuyos objetivos son los siguientes:

a. Analizar el plan de estudios vigente para determinar los espacios formativos, subcompetencias y contenidos relacionados con la competencia de atención a la diversidad, en las áreas curriculares de formación de fundamentos pedagógicos y formación pedagógica didáctica orientada.

b. Analizar las necesidades formativas del personal docente de la UPNFM, para la concreción en el aula de la competencia genérica de atención a la diversidad.

c. Identificar las percepciones del estudiantado sobre el desarrollo de la competencia de atención a la diversidad, durante el proceso formativo en la UPNFM.

d. Diseñar, a partir de las necesidades identificadas, un manual para el desarrollo de la competencia genérica de atención a la diversidad. 


\section{Sustento teórico}

Esta investigación se centra en el estudio de la formación inicial de docentes y la competencia genérica de reconocimiento y aprecio de la diversidad humana. Sánchez (2008) explica:

Las competencias transversales, también conocidas como genéricas, son un grupo de competencias que nacen de la intersección entre las diferentes disciplinas. Estas competencias no están directamente ligadas a ninguna disciplina, sino que pueden ser aplicadas a una variedad de áreas de materia y situaciones. (p. 9)

Perrenoud (1997, citado por Sánchez, 2008) enfatiza:

Las competencias transversales son parte de las características generales de la acción humana, las que principalmente se describen sobre la "actuación comunicacional" y "la acción técnica". A partir de estas características generales de la acción humana, Perrenoud P. presenta tres calificativos para éstas:

- Interactivas (se relacionan con las normas sociales, relacionales y transaccionales),

- Simbólicas (uso del lenguaje y también sistemas de códigos e iconos),

- No programadas (requiere de la toma de decisión individual). (p. 9)

En este sentido, la preparación del personal docente en pre-servicio deberá estar unificada a una serie de procesos reflexivos que permitan constantemente revisar las visiones sobre la enseñanza, el aprendizaje, y las diferencias individuales (Paz, 2014). El desarrollo de una competencia docente, vinculada con el reconocimiento de la diversidad humana como valor, tiene como propósito la mejora de las instituciones educativas y la creación de culturas inclusivas, eliminando los procesos excluyentes de la educación, consecuencia del prejuicio y de concepciones segregacionistas de las diferencias humanas (Messiou et al., 2016).

Hay que mencionar además que la formación de este tipo de competencia contribuirá con la confianza, el gusto por la realización de las tareas docentes y la identidad profesional, ¿qué y quiénes son?, su autoimagen, los significados que vinculan a sí y a su trabajo, sus relaciones con el alumnado, sus papeles y las conexiones entre este y su vida fuera de la escuela (Day, 2006; Kelchtermans, 1993).

Para la European Commission (2017), la formación inicial docente para la diversidad implica aumentar la comprensión del mundo y de sus culturas. La necesidad de desarrollar competencias de comunicación para la diversidad, así como la capacidad de formar profesorado empático y reflexivo acerca de sus propias creencias, diferencias culturales y socioeconómicas. De igual forma para crear escuelas efectivas y de calidad los maestros y las maestras deben prepararse para aprovechar los beneficios de la diversidad de sus estudiantes, este tipo de formación inicial con el reconocimiento de la diversidad humana, aspecto que puede contribuir con la enseñanza y la mejora de la formación docente (Ragoonaden, Sivia \& Baxan, 2015). 
doi: http://dx.doi.org/10.15359/ree.21-3.19

URL: http://www.una.ac.cr/educare

CORREO: educare@una.cr

\section{Método}

\section{Tipo de estudio}

Toda investigación está orientada por los objetivos planteados durante la delimitación del objeto de estudio. En ellos se plasma el propósito y se establecen las bases para la construcción de la estrategia metodológica a seguir, la cual estará fuertemente influenciada por la intencionalidad de quien investiga.

Este proyecto de investigación se ha desarrollado desde la perspectiva de la investigación evaluativa, aplicando metodología cualitativa, ya queesta clase de métodos busca la comprensión de la conducta humana desde el propio marco de referencia de quien actúa (Bisquerra, 2004). Es decir, la perspectiva que se asume es "desde dentro" y se orienta al descubrimiento, explorando y describiendo el fenómeno sujeto a estudio. También se ha empleado metodología de corte cuantitativo, en específico el diseño de tipo encuesta, de corte transversal, pues la medición se efectuó en un único momento, sin aplicaciones posteriores.

\section{Población y muestra}

La investigación se ha llevado a cabo teniendo como escenario el Sistema Presencial de la Universidad Pedagógica Nacional Francisco Morazán UPNFM, institución del Estado de Honduras responsable de la formación de docentes a nivel nacional. La UPNFM ofrece veintitrés carreras con varias orientaciones en el nivel de pregrado, así como trece maestrías y un doctorado en educación.

En relación con los sujetos participantes se han considerado dos poblaciones importantes, la primera está formada por un grupo de ocho docentes y la segunda por una muestra de 225 estudiantes de Práctica Profesional, quienes estarían por culminar su formación universitaria de pregrado; con la finalidad de contar con las opiniones de los dos actores principales del proceso formativo.

\section{Técnicas e instrumentos para recolección de datos}

Para la recolección de información se emplearon, como técnicas, el cuestionario, el cual se aplicó a los 225 estudiantes que componen la muestra del estudio y la entrevista semiestructurada que sirvió para recolectar las opiniones y experiencias docentes en relación con el desarrollo de la competencia en estudio a nivel de aula. Para complementar la información se hizo uso del análisis documental de los planes de estudio vigentes.

Para cumplir con los requisitos de validez y confiabilidad se sometió el cuestionario a un estudio piloto, el Alpha de Cronbach=0.83 demostró la consistencia interna de los ítems del instrumento. La guía de preguntas para la entrevista fue sometida a una revisión de personas expertas; posterior a sus observaciones, se readecuaron para obtener la versión final. 


\section{Resultados}

Los resultados de este estudio se presentan por cada uno de los objetivos de investigación.

\section{Análisis al plan de estudios}

La competencia de reconocimiento y aprecio por la diversidad se entiende en el plan de estudios como: la capacidad de comprender y aceptar la diversidad física, psicológica, social y cultural, como un componente enriquecedor personal y colectivo, con el fin de promover la convivencia en paz entre las personas, sin incurrir en distinciones de género, edad, religión, etnia, condición social y política (Aguilar, 2007).

En los planes de estudio (UPNFM, 1994), la formación docente se realizaba desde un enfoque curricular por objetivos y asignaturas fragmentadas, centradas en los procesos de enseñanza. Estos planes y programas se mantuvieron vigentes hasta el año 2007, en el que se inicia un proceso de reforma curricular denominado Planes 2008 (UPNFM, 2008) desde la perspectiva de las competencias académico profesionales del proyecto Tunning Latinoamérica. Para valorar los cambios en materia de formación para la atención a la diversidad, se revisó la estructura curricular entre ambos planes (Véase Tabla 1).

Esta revisión demostró que las asignaturas del Plan 1994 (UPNFM, 1994), vinculadas con contenidos relativos al reconocimiento y aprecio por la diversidad humana, carecían de un componente orientado a desarrollar esta competencia, a excepción de la asignatura denominada Desarrollo Curricular que abordaba el estudio de la adaptación del currículo hacia la atención de las diferencias individuales del estudiantado, dejando de lado aquellas diferencias derivadas de la pertenencia a un grupo cultural, social o lingüístico.

En cuanto al Plan 2008(UPNFM, 2008), hay una marcada intención de promover capacidades docentes propias de la educación inclusiva, buscando el reconocimiento de la diversidad humana como un valor y recurso para la creación de experiencias de aprendizaje en contextos de calidad y equidad, incluyendo subcompetencias tales como 'promover actitudes que aseguren una vida digna a las clases y grupos sociales más desfavorecidos y con necesidades especiales'.

Por lo tanto, a nivel de diseño curricular es posible concluir que se ha logrado un avance significativo en materia de formación docente para atención a la diversidad, ya que la reforma 2008, incorporó al plan de estudios elementos que permiten reconocer el valor educativo de la diversidad.

Es importante destacar que, aunque se ha efectuado este avance, es necesario incluir otras temáticas y experiencias prácticas, para que en el futuro cada docente cuente con herramientas básicas, que no solo le permitan apreciar y reconocer la diversidad, sino que le conduzcan a brindar una respuesta inclusiva a las diferencias del alumnado; entre ellas podemos mencionar: la adaptación de la enseñanza, la acción tutorial, el análisis y reflexión sobre el prejuicio y la exclusión. 
doi: http://dx.doi.org/10.15359/ree.21-3.19

URL: http://www.una.ac.cr/educare

CORREO: educare@una.cr

Tabla 1: Comparación de la estructura curricular orientada a la atención de la diversidad entre el Plan 94 y Plan 2008.

\begin{tabular}{|c|c|c|c|c|}
\hline \multicolumn{2}{|r|}{ Plan 1994} & \multicolumn{3}{|c|}{ Plan 2008} \\
\hline Asignatura & Contenidos & $\begin{array}{l}\text { Espacio } \\
\text { pedagógico }\end{array}$ & Subcompetencias & Temáticas \\
\hline Pedagogía & $\begin{array}{l}\text { Teorías, modelos y componentes } \\
\text { pedagógicos. } \\
\text { La pedagogía como sistema. }\end{array}$ & Pedagogía & $\begin{array}{l}\text { Mostrar respeto y tolerancia hacia } \\
\text { los demás valorando las diferentes } \\
\text { opiniones y posturas como una } \\
\text { oportunidad de enriquecimiento } \\
\text { personal y trabajo en equipo. }\end{array}$ & $\begin{array}{l}\text { La interculturalidad y la } \\
\text { diversidad en el aula de } \\
\text { clase } \\
\text { Educación para una } \\
\text { cultura de paz }\end{array}$ \\
\hline Sociología & $\begin{array}{l}\text { Los contenidos estaban } \\
\text { orientados al estudio del } \\
\text { pensamiento sociológico } \\
\text { y teorías del desarrollo } \\
\text { socioeconómico. }\end{array}$ & Sociología & $\begin{array}{l}\text { Capacidad para identificar el fenómeno } \\
\text { de la desigualdad social con el propósito } \\
\text { de transformar las condiciones de vida y } \\
\text { de convivencia humana. } \\
\text { Promover actitudes que aseguren } \\
\text { una vida digna a las clases y grupos } \\
\text { sociales más desfavorecidos y con } \\
\text { necesidades especiales. }\end{array}$ & $\begin{array}{l}\text { - Dinámica y estructura de } \\
\text { la diferenciación social en } \\
\text { la sociedad hondureña } \\
\text { - Teoría del desarrollo y } \\
\text { subdesarrollo de América } \\
\text { Latina } \\
\text { - Ambiente y sociedad. } \\
\text { - Estructuras sociales }\end{array}$ \\
\hline $\begin{array}{l}\text { Desarrollo } \\
\text { Curricular }\end{array}$ & $\begin{array}{l}\text { Diseño de planes y programas } \\
\text { de estudio, su relación con las } \\
\text { diferencias individuales y con los } \\
\text { recursos educativos disponibles. }\end{array}$ & $\begin{array}{l}\text { Diseño y } \\
\text { desarrollo } \\
\text { curricular }\end{array}$ & $\begin{array}{l}\text { Realizar adecuaciones curriculares } \\
\text { atendiendo las características del } \\
\text { contexto y de los participantes. }\end{array}$ & - Currículo y diversidad \\
\hline $\begin{array}{l}\text { Didáctica } \\
\text { General }\end{array}$ & $\begin{array}{l}\text { Planificación didáctica y } \\
\text { metodología de enseñanza. }\end{array}$ & $\begin{array}{l}\text { Didáctica de los } \\
\text { aprendizajes }\end{array}$ & $\begin{array}{l}\text { Adecuar los diferentes recursos } \\
\text { didácticos y sus posibilidades de } \\
\text { aplicación a los diferentes contextos, } \\
\text { promoviendo el respeto a la } \\
\text { diversidad y multiculturalidad } \\
\text { Mostrar respeto y tolerancia hacia } \\
\text { las demás personas valorando las } \\
\text { diferentes opiniones y posturas como } \\
\text { una oportunidad de enriquecimiento } \\
\text { personal y trabajo en equipo. }\end{array}$ & $\begin{array}{l}\text { Estrategias didácticas } \\
\text { según especialidad. } \\
\text { Proceso de planificación } \\
\text { didáctica }\end{array}$ \\
\hline $\begin{array}{l}\text { Didáctica } \\
\text { Especial }\end{array}$ & $\begin{array}{l}\text { Elaboración de recursos de } \\
\text { aprendizaje y desarrollo de } \\
\text { clases. }\end{array}$ & $\begin{array}{l}\text { Práctica } \\
\text { profesional I }\end{array}$ & $\begin{array}{l}\text { Saber adaptarse a la pluralidad y } \\
\text { heterogeneidad de los grupos. }\end{array}$ & $\begin{array}{l}\text { Situación actual de } \\
\text { la educación frente a } \\
\text { contextos educativos y } \\
\text { sociales específicos }\end{array}$ \\
\hline $\begin{array}{l}\text { Práctica } \\
\text { Docente }\end{array}$ & $\begin{array}{l}\text { Proceso general de la práctica } \\
\text { docente, su organización, } \\
\text { diagnóstico y planificación. }\end{array}$ & $\begin{array}{l}\text { Práctica } \\
\text { profesional II }\end{array}$ & $\begin{array}{l}\text { Diseñar, implementar y evaluar } \\
\text { estrategias de enseñanza aprendizaje } \\
\text { adaptadas según aptitud, interesas, } \\
\text { rendimientos, conocimientos previos, } \\
\text { necesidades espaciales y naturaleza } \\
\text { de la disciplina. } \\
\text { Planificar y operacionalizar estrategias } \\
\text { de enseñanza aprendizaje según } \\
\text { los contextos, niveles y desarrollo } \\
\text { psicobiosocial del estudiantado. }\end{array}$ & $\begin{array}{l}\text { En este espacio } \\
\text { pedagógico no se } \\
\text { desarrollan contenidos } \\
\text { conceptuales, pero } \\
\text { sí se trabajan los } \\
\text { procedimentales y } \\
\text { actitudinales. }\end{array}$ \\
\hline
\end{tabular}

Nota: Estos resultados se han obtenido a partir del análisis de los Planes de estudio de pregrado (UPNFM, 1994) y los Planes de estudio de pregrado (UPNFM, 2008). 
En cuanto a las estrategias didácticas y de evaluación para promover la competencia, se declaran algunas que han demostrado su efectividad durante la formación, sin embargo, se pueden incorporar e implementar el estudio de caso, el aprendizaje en servicio, actividades reflexivas, que vengan a fortalecer la formación del profesorado para la diversidad.

Durante la revisión fue posible identificar que el espacio formativo de Evaluación de los Aprendizajes no incluye la competencia. Considerando que la evaluación también debe responder a la diversidad humana en educación, sería deseable incluir saberes orientados a la inclusión y la atención a la diversidad.

\section{Identificación de necesidades formativas en el profesorado universitario}

La consulta a docentes por medio de la entrevista semi- estructurada, incluía tres secciones: a) conocimientos sobre la diversidad humana y sus implicaciones educativas; b) estrategias didácticas utilizadas para el desarrollo de la competencia en estudio y c) dificultades identificadas para la formación en materia de diversidad e inclusión. Los resultados permitieron conocer las percepciones de los actores, acerca del trabajo en las aulas en el desarrollo de la competencia de reconocimiento y aprecio por la diversidad.

Posterior al proceso de análisis de contenido de las entrevistas se identificaron dos tendencias principales que se describen a continuación. (Véase Figura 1)

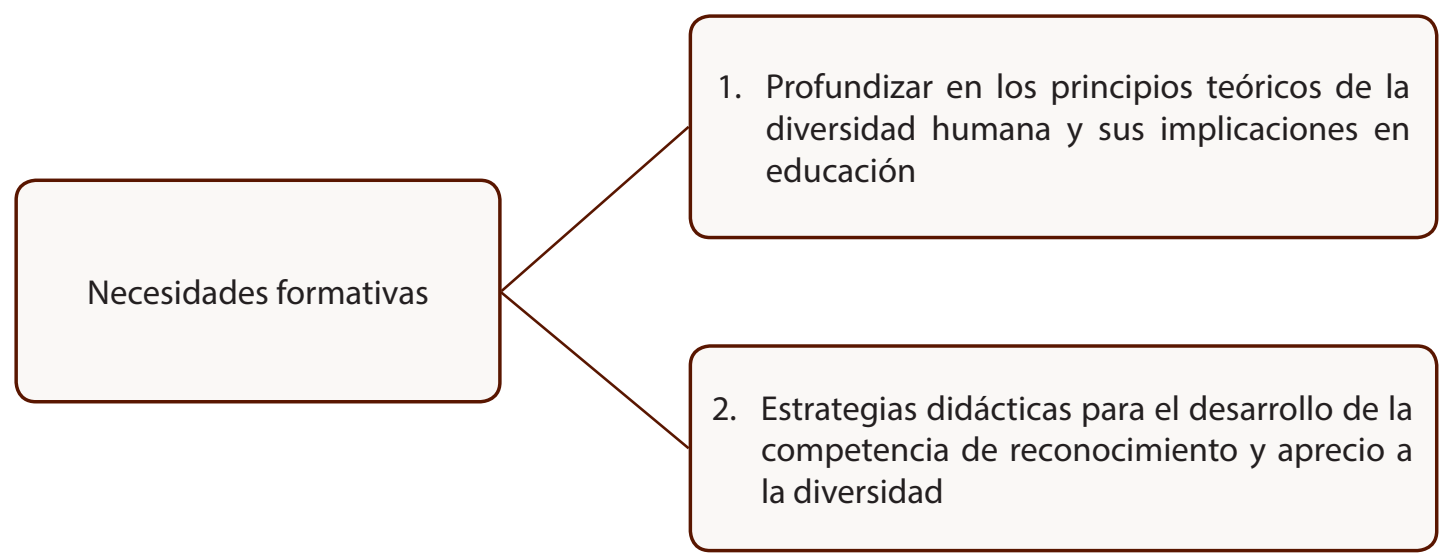

Figura 1: Necesidades formativas de los docentes y las docentes para desarrollar la competencia de diversidad.

Aunque se cuenta con saberes básicos sobre la diversidad, los docentes y las docentes manifiestan la necesidad de contar con más y mejores conocimientos sobre la diversidad humana y su significado en la educación. La mayoría identifica conocimientos relacionados con las diferencias individuales y de grupo, dentro de estos se destacan la cultura y la clase social. 
doi: http://dx.doi.org/10.15359/ree.21-3.19

URL: http://www.una.ac.cr/educare

CORREO: educare@una.cr

Hay que reconocer que sólo tengo un poco de conocimiento sobre estos tópicos por eso se hace difícil desarrollar algo que uno no sabe. Docente 03

De igual manera se manifiesta dificultad en el desarrollo de la competencia por falta de conocimiento de estrategias didácticas propias de esta clase de formación. Esta situación se torna más compleja en clases numerosas, tal y como expresa una docente del espacio formativo de psicología de los aprendizajes:

Es difícil, dentro de las clases generales, desarrollar esta competencia, dado que son clases extremadamente numerosas, de entre setenta a ochenta estudiantes, y se exige de parte de la universidad -porque dentro de la evaluación que se nos hace al final de la asignatura o del espacio pedagógico mejor dicho se nos exige que llevemos un ritmo de trabajo dentro de un contenido curricular- sin embargo, este contenido curricular no ha sido muy tomado en cuenta. Docente 07

\section{Percepciones del estudiantado sobre el desarrollo de la competencia}

En un primer momento se consultó al estudiantado sobre el concepto de diversidad humana en educación. El $63.1 \%$ de participantes cuenta con una concepción amplia acerca de la diversidad, entendida esta como las diferencias individuales y de grupo, que traen consigo implicaciones educativas, que merecen una respuesta incluyente. Este porcentaje muestra una tendencia favorable en el reconocimiento de la diversidad como un valor pedagógico. El resto del estudiantado considera solo un aspecto de la diversidad, como la población de personas con necesidades educativas especiales o los diversos estilos de aprendizaje, lo que indica una visión reduccionista y orientada a las diferencias derivadas de la individualidad (Véase Tabla 2).

Tabla 2: Conceptualización sobre diversidad humana en educación del estudiantado participante en el estudio

Conceptualización de diversidad humana en educación

- Diversos estilos de aprendizaje

- Estudiantes provenientes de culturas diversas

- La diversidad de métodos de enseñanza

- Personas con necesidades especiales

- Las diversas capacidades individuales, culturales, lingüísticas, de género que deben atenderse en el aula 142

\section{f $\%$}

$21 \quad 9.3$

$8 \quad 3.6$

$16 \quad 7.1$

$38 \quad 16.9$

63.1

Total

225100

10 
En cuanto a los saberes conceptuales relacionados con la diversidad, ver Tabla 3, el tópico sobre las inteligencias múltiples es el más abordado (57.3\%), le siguen las metodologías en el aula para atender la diversidad (48.9\%) y los estilos y ritmos de aprendizaje (45.8\%). En menor grado de estudio, se encuentran temáticas como educación intercultural (20.9\%), pedagogía de la diversidad (15.1\%) y medidas de atención a la diversidad (14.2\%). Lo anterior indica un fuerte énfasis en los tópicos relacionados con las diferencias individuales derivadas de las condiciones biológicas y cognitivas del estudiantado; sin embargo, aún se identifican debilidades en el reconocimiento de la diversidad a nivel de grupo, así como las medidas de atención a las diferencias de estudiantes, con el desafío de consolidar el aprendizaje de saberes conceptuales

Tabla 3: Saberes conceptuales relacionados con la diversidad humana

\begin{tabular}{lcc}
\hline Saberes conceptuales adquiridos & $\mathrm{f}$ & $\%$ \\
\hline - Teoría de las inteligencias múltiples & 129 & 57.3 \\
- Estilos y ritmos de aprendizaje & 103 & 45.8 \\
- Capacidades intelectuales diversas & 85 & 37.8 \\
- Adaptaciones curriculares & 77 & 34.2 \\
- Metodologías en el aula para atender la diversidad & 110 & 48.9 \\
- Pedagogía de la diversidad & 34 & 15.1 \\
- Ámbitos de la diversidad del estudiantado & 45 & 20.0 \\
- Medidas de atención a la diversidad & 32 & 14.2 \\
- Acción tutorial & 54 & 24.0 \\
• Educación intercultural & 47 & 20.9 \\
\hline Total & 225 & 100 \\
\hline
\end{tabular}

en este campo.

La formación inicial de docentes en el ámbito de la atención a la diversidad requiere de habilidades específicas de actuación. En estesentido, se indagósobre el desarrollo decapacidades propias de la educación inclusiva. Los resultados indican que el 60\% del estudiantado es capaz de reconocer la diversidad. Posteriormente, menos de la mitad manifestó contar con habilidades para proponer estrategias de atención según las inteligencias múltiples.

Solo un $28 \%$ considera haber desarrollado capacidades para el desarrollo de la educación intercultural. Estos resultados ponen en evidencia la necesidad de fortalecer la formación 
doi: http://dx.doi.org/10.15359/ree.21-3.19

URL: http://www.una.ac.cr/educare

CORREO: educare@una.cr

Tabla 4: Saberes procedimentales adquiridos por el estudiantado

\begin{tabular}{lrc}
\hline \multicolumn{1}{c}{ Saberes procedimentales adquiridos } & $\mathrm{f}$ & $\%$ \\
\hline - Reconocer la diversidad en el estudiantado & 135 & 60.0 \\
- Adaptar el currículo & 98 & 43.6 \\
- Estrategias de acción tutorial & 100 & 44.4 \\
- Trabajo en equipo interdisciplinario & 81 & 36.0 \\
- Elaboración de estrategias de aprendizajes según ritmo y estilos del estudiantado & 72 & 32.0 \\
- Experiencias de aprendizaje para favorecer las inteligencias múltiples & 110 & 48.9 \\
- Experiencias de aprendizaje desde la educación & 63 & 28.0 \\
\hline Total & 225 & 100 \\
\hline
\end{tabular}

docente para y en la diversidad, haciendo especial énfasis en el trabajo colaborativo, las adaptaciones curriculares y la educación intercultural (Véase Tabla 4).

\section{Discusión}

Desde que la respuesta educativa a las diferencias dio un giro hacia la educación inclusiva, se ha gestado la necesidad de incorporar a los planes de estudio de las carreras de magisterio, formación para atender las necesidades educativas de todo el estudiantado.

En el caso de la UPNFM, de manera reciente, se incluyó en los planes de estudio una competencia genérica o transversal, que está orientada al reconocimiento y aprecio por la diversidad, los investigadores sugieren que esta formación se debe constituir en un eje transversal, ya que solo de esta manera es posible superar los conocimientos fraccionados (Aksamit, 1990; Blanton et al. 1996; Cook, 2002). En este sentido, al realizar el análisis a los planes de estudio, se identificó que la competencia ha sido declarada de forma transversal en las áreas curriculares de Formación de Fundamentos Pedagógicos y Formación Pedagógica Didáctica Orientada, lo que constituye la oportunidad para desarrollar el reconocimiento y aprecio de la diversidad como un elemento valioso para la educación.

El informe de la European Agency for Development in Special Needs Education (2011) detalla que las cuestiones relacionadas con la diversidad y la inclusión son tratadas más frecuentemente en los cursos de educación especial que en los cursos generales destinados a todos los docentes y las docentes. Quienes escriben este artículo reconocen que puede haber un peligro en la incorporación de temas de inclusión en todos los cursos, con el riesgo de que en el impacto se reduzca o se pierda por completo el enfoque, por lo que será importante que 
el pensum ${ }^{6}$ incluya una asignatura específica al respecto, como señala la investigación de Paz, (2014) en el contexto de la UPNFM.

La formación para la inclusión educativa no puede limitarse a ciertos ámbitos de las diferencias humanas; al contrario, debe abordar las cuestiones sobre la diversidad de manera más amplia (Gultig, 1999). En el caso de la UPNFM, la formación está más orientada a las diferencias individuales que tienen que ver con los ritmos y estilos de aprendizaje, las de capacidad intelectual, la discapacidad, y deja por fuera el género o la personalidad, por ejemplo. En cuanto al estudio de las diferencias de grupo son casi inexistentes los temas que se incluyen durante la formación, aspectos como la cultura, el grupo social, la lengua no son tomados en cuenta. Este es, en especial, un aspecto a mejorar dado que el contexto hondureño se caracteriza por su alta heterogeneidad cultural, lingüística y social.

Existe consenso en que la inclusión educativa de estudiantes no puede realizarse sin una decidida intervención de los docentes. Para que esto sea posible, es necesario ubicar su centralidad en la educación y demostrar que no se puede avanzar en este plano sin mejorar, al mismo tiempo, en la comprensión de lo que cree, puede hacer y hace el docente. (Calvo, 2013, p. 6)

Para que la formación en atención a la diversidad sea una realidad, se requiere que las personas formadoras de personal formador se apropien de los principios teóricos y metodológicos de esta clase de respuesta educativa, tal y como expresa el grupo de docentes que han participado en esta investigación.

Schmelkes (2013) explica que en este tiempo debemos aprender a ver el mundo como un complejo de heterogeneidades y no como un todo homogéneo. Reconocer que nos movemos en un escenario donde la diversidad está en todas las latitudes; las soluciones no pueden ser iguales para unas realidades diversas y diferentes. La complejidad implícita en la comprensión y en la búsqueda de soluciones se convierte en uno de los principales requisitos hoy día. En ese sentido, formar para la diversidad y en la diversidad requiere un proceso de desarrollo profesional del personal docente universitario en la UPNFM, que fortalezca el trabajo de las redes académicas mediante procesos de investigación acción reflexión.

Un docente necesita, ante todo, capacidad de construirse un punto de vista personal que implica una identidad propia, como persona capaz de aprender, de ser responsable e innovadora, así como capacidad para resolver tareas estableciendo sus propias metas, planteando sus propias estrategias, procesando información y encontrando recursos para aprender. También debe ser capaz de flexibilizar el currículum para poder contextualizarlo y garantizar la permanencia de los estudiantes en el centro educativo, además de priorizar

${ }^{6}$ Plan de estudios de una carrera que da a conocer las materias o asignaturas que se estudiarán en cada período de la misma. 
doi: http://dx.doi.org/10.15359/ree.21-3.19

URL: http://www.una.ac.cr/educare

CORREO: educare@una.cr

el trabajo entre la escuela y la comunidad. (Calvo, 2013, p. 9)

Sin embargo, tal y como se identificó en un estudio realizado por el CERI (2010), el personal docente formador de las nuevas generaciones formadoras deberá emplear las estrategias didácticas pertinentes con la formación para la diversidad, señalamiento que han efectuado las personas docentes participantes en esta investigación.

Uno de los elementos fundamentales para atender la diversidad del estudiantado desde ambientes inclusivos es el reconocimiento y aprecio por las diferencias humanas. En el caso del estudiantado participante de este estudio, se identificó que más del $60 \%$ contaba con un concepto amplio y claro de la diversidad, aspecto que coincide con un estudio anterior realizado en el contexto de la UPNFM (Paz, 2014). Respecto a los saberes que ha adquirido el estudiantado, encontramos que, a nivel conceptual, se encuentran potenciados los conocimientos relativos a las diferencias individuales como las inteligencias múltiples, o los ritmos y estilos de aprendizaje. A nivel procedimental el $60 \%$ dijo sentirse en la capacidad de reconocer la diversidad, pero menos del 30\% indicó tener habilidades para adecuar el currículo, atender la diversidad cultural

\begin{tabular}{|l|l|}
\cline { 2 - 2 } Teóricos & $\begin{array}{l}\text { Reconocimiento de la } \\
\text { diversidad humana (Cardona, } \\
\text { 2006; Wang, 1999). }\end{array}$ \\
\cline { 2 - 2 } & Inclusión (Ainscow, 2001) \\
\hline $\begin{array}{l}\text { Formación inicial de } \\
\text { docentes para atender la } \\
\text { diversidad en contextos } \\
\text { inclusivos (Duck, 2003: } \\
\text { Moriña, 2008). }\end{array}$ \\
\hline
\end{tabular}

\begin{tabular}{l|l|}
\cline { 2 - 2 } Empíricos & $\begin{array}{l}\text { Perfil de competencias } \\
\text { docentes para la inclusión. } \\
\text { (EADSNE, 2011). }\end{array}$ \\
\cline { 2 - 2 } & $\begin{array}{l}\text { Respuesta del profesorado } \\
\text { frente a la diversidad (CERI, } \\
\text { 2010). }\end{array}$ \\
\hline $\begin{array}{l}\text { Modelo de formación } \\
\text { docente para atender la } \\
\text { diversidad (Van Laarhoven et } \\
\text { al., 2007). }\end{array}$ \\
\hline
\end{tabular}

Figura 2: Sustento teórico y empírico del manual para transversalizar la competencia en estudio.

o trabajar en equipo, estos son considerados aspectos que se deben incluir en la formación en inclusión (Carroll, Forlin, y Jobling, 2003).

Los resultados de este proceso diagnóstico han propiciado la necesidad de diseñar una herramienta que contribuya a desarrollar la competencia. Se consideró que un manual reunía las características necesarias para los propósitos establecidos. Posteriormente se consultaron 
referentes teóricos y empíricos que dieran sustento al documento (Véase Figura 2).

El manual está estructurado en tres elementos principales, el primero de ellos es la conceptualización de la competencia de diversidad. Un segundo aspecto está referido a la desagregación de la competencia en los cuatro niveles de logro, incluyendo una propuesta de transversalización de esta misma en los espacios de Formación de Fundamentos Pedagógicos y Formación Pedagógica Didáctica Orientada. El tercer aspecto ofrece las estrategias didácticas y de evaluación que se proponen para el desarrollo de la competencia.

\section{Conclusiones}

- Los planes de estudio de las carreras de la UPNFM tienen declarada la competencia de reconocimiento y aprecio por la diversidad en la Formación de Fundamento Pedagógico y en la Orientada: este es un aspecto positivo. Sin embargo, pareciera que la concreción de este eje transversal se diluye y no termina de llegar a la formación del estudiantado. La formación para atender la diversidad debe realizarse no solamente desde la concreción de un tema transversal. La bibliografía empírica ha demostrado la importancia de contar con un espacio formativo orientado a conocer la diversidad humana, implicaciones educativas y su respuesta inclusiva, por lo que sería deseable incorporar a una asignatura específica a los planes de estudio.

- Para el desarrollo de esta competencia el personal docente participante opina que se hacen necesarios conocimientos más claros sobre la diversidad, de estrategias didácticas y de evaluación pertinentes con la formación para la educación inclusiva. Para favorecer el desarrollo profesional del personal formador de docentes, se debe implementar un trabajo colegiado en el desarrollo de estrategias para concretar la formación para la diversidad. El manual para el desarrollo de la competencia de Atención a la diversidad es un medio para cumplir este fin.

- Si bien es cierto la formación ha permitido al estudiantado contar con un concepto amplio de la diversidad, aún no se han logrado conocimientos de las diferencias de grupo, ni de habilidades para adaptar el currículo, trabajar en equipo, así como estrategias para atender las diferencias culturales. En cuanto a las actitudes se identifican como favorables con una respuesta a la diversidad de naturaleza incluyente.

- El manual para el desarrollo de la Competencia de atención a la diversidad es una herramienta elaborada a partir de los principios teóricos de la diversidad y de referentes empíricos sustantivos en la línea de investigación de la formación docente para la inclusión educativa.

\section{Referencias}


doi: http://dx.doi.org/10.15359/ree.21-3.19

URL: http://www.una.ac.cr/educare

CORREO: educare@una.cr

Aguilar, L. (2007). Documento base reforma curricular Universidad Pedagógica Nacional Francisco Morazán. Tegucigalpa: UPNFM.

Ainscow, M. (2001). Desarrollo de escuelas inclusivas: Ideas, propuestas y experiencias para mejorar las instituciones escolares. Madrid: Narcea Ediciones.

Aksamit, D. L. (1990). Practicing teachers' perceptions of their preservice preparation for mainstreaming. Teacher Education and Special Education: The Journal of the Teacher Education Division of the Council for Exceptional Children, 13(1), 21-29. doi: https://doi. org/10.1177/088840649001300104

Artiles, A. J. (1998). The dilemma of difference. Enriching the disproportionality discourse with theory and context. The Journal of Special Education, 32(1), 32-36. doi: https://doi. org/10.1177/002246699803200105

Ball, A. F., y Tyson, C. A. (Eds.). (2011). Studying diversity in teacher education. Maryland: AERA.

Bisquerra, R. (2004). Metodología de la investigación educativa. Madrid: La Muralla

Blanton, L. P., Griffin, C. C., Winn, J. A. y Pugach, M. C. (1996). Teacher education in transition collaborative programs to prepare general and special educators. Denver: Love.

Calvo, G. (2013). La formación de docentes para la inclusión educativa. Páginas de Educación, 6(1), 1-22. Recuperado de http://www.scielo.edu.uy/pdf/pe/v6n1/v6n1a02.pdf

Cardona, M. C. (2006). Diversidad y educación inclusiva: Enfoques metodológicos y estrategias para una enseñanza colaborativa. Madrid: Pearson.

Carroll, A., Forlin, C., \& Jobling, A. (2003). The impact of teacher training in special education on the attitudes of Australian preservice general educators towards people with disabilities. Teacher Education Quarterly, 30(3), 65-79. Recuperado de http://files.eric. ed.gov/fulltext/EJ852365.pdf

Centre for Educational Research and Innovation (CERI). (2010). Educating teachers for diversity: Meeting the challenge. París: OECD. doi: 10.1787/ 20769679

Cook, B.G. (2002). Inclusive attitudes, strengths, and weaknesses of pre-service general educators enrolled in a curriculum infusion teacher preparation program. Teacher Education and Special Education: The Journal of the Teacher Education Division of the Council for Exceptional Children, 25(3), 262-277. doi: https://doi.org/10.1177/088840640202500306

Day, C. (2006). Pasión por enseñar: La identidad personal y profesional del docente y sus valores. Madrid: Narcea Ediciones. 
De Valle, A. y Vega, V. (2006). Una escuela en y para la diversidad: El entramado de la diversidad. Buenos Aires: Aique Editores.

Duck, C. (Ed.). (2003). Educar en la diversidad: Material de formación docente. Santiago de Chile: UNESCO.

European Agency for Development in Special Needs Education. (EADSNE). (2011). Formación del profesorado para la educación inclusiva en Europa: Retos y oportunidades. Odense, Dinamarca: Autor. Recuperado de https://www.european-agency.org/sites/default/files/ te4i-challenges-and-opportunities TE4I-Synthesis-Report-ES.pdf

European Commission. (2017). Preparing teachers for diversity: The role of initial teacher education. Brussels: Publications Office of the European Union. Recuperado de http://emn.ie/files/p 20170608124500PreparingTeachersforDiversity.pdf

García, C. (2005). Educación y diversidad. Málaga: Aljibe.

Gultig, J. (1999). Rethinking the role of theory and practice in South African teacher education. Perspectives in Education, 18(1), 55-80.

Kelchtermans, G. (1993). Getting the story, understanding the lives: From career stories to teacher's professional development. Teaching ad Teacher Education, 9(5-6), 443-456. doi: https://doi.org/10.1016/0742-051X(93)90029-G

Luque, A. y Delgado, C. M. (2002). Diversidad humana y educación: Intervenciones para optimizar el desarrollo psicológico. Intervención Psicosocial, 11(2), 143-165. Recuperado de http:// www.copmadrid.org/webcopm/publicaciones/social/78067.pdf

Moriña, A. (2008). La escuela de la diversidad: Materiales de formación para el profesorado. Madrid: Editorial Sintesis.

Messiou, K., Ainscow, M., Echeita, G., Goldrick, S., Hope, M., Paes, I., ... Vitorino, T. (2016). Learning from differences: A strategy for teacher development in respect to student diversity. School Effectiveness and School Improvement, 27(1), 45-61. doi: https://doi.org/10.1080/0 $\underline{9243453.2014 .966726}$

Paz, C. L. (2006). Diagnóstico y propuesta para el desarrollo de competencias docentes para la atención a estudiantes con discapacidad a nivel universitario (Tesis de maestría). Universidad Pedagógica Nacional Francisco Morazán. Tegucigalpa, Honduras. Recuperado de http://www.cervantesvirtual.com/obra/diagnostico-y-propuesta-para-el-desarrollode-competencias-docentes-para-la-atencion-a-estudiantes-con-discapacidad-a-niveluniversitario/

Paz, C. L. (2014). Competencias docentes para la atención a la diversidad: Investigación acción en 
doi: http://dx.doi.org/10.15359/ree.21-3.19

URL: http://www.una.ac.cr/educare

CORREO: educare@una.cr

la Universidad Pedagógica Nacional Francisco Morazán de Honduras (Tesis de doctorado). Universidad de Alicante, España.

Ragoonaden, K. O., Sivia, A., \& Baxan, V. (2015). Teaching for diversity in teacher education: Transformative frameworks. The Canadian Journal for the Scholarship of Teaching and Learning, 6(3), 1-18. doi: https://doi.org/10.5206/cjsotl-rcacea.2015.3.6

Sánchez, A. (2008). Competencias transversales: Una mirada actual. Revista CoLearn, 1(1), 8-10. Recuperado de: http://www.open.edu/openlearnworks/pluginfile.php/54151/mod resource/content/1/REVISTA COLEARN 1.pdf

Schmelkes, S. (2013). Educación para un México intercultural. Sinéctica, 40, 1-12. Recuperado de http://www.scielo.org.mx/pdf/sine/n40/n40a2.pdf

Thomas, G. y Loxley, A. (2007). Deconstructing special education and constructing inclusion. Buckingham: McGraw-Hill International.

Unesco. (1990). Declaración mundial sobre educación para todos y marco de acción para satisfacer las necesidades básicas de aprendizaje. Jomtiem, Tailandia: Autor. Recuperado de http:// www.unesco.org/education/pdf/JOMTIE S.PDF

Unesco. (1994). Declaración de Salamanca y marco de acción para las necesidades educativas especiales. Salamanca: Autor. Recuperado de http://www.unesco.org/education/pdf/ SALAMA S.PDF

Universidad Pedagógica Nacional Francisco Morazán (UPNFM). (1994). Planes de estudio de pregrado. Tegucigalpa, Honduras: Autor.

Universidad Pedagógica Nacional Francisco Morazán (UPNFM). (2008). Planes de estudio de pregrado. Tegucigalpa, Honduras: Autor.

Van Laarhoven, T. R., Munk, D. D., Lynch, K., Bosma, J., \& Rouse, J. (2007). A model for preparing special and general education preservice teachers for inclusive education. Journal of Teacher Education, 58(5), 440-455. doi: https://doi.org/10.1177/0022487107306803

Wang, M. C. (1999). Atención a la diversidad del alumnado (3ª ed.). Madrid: Narcea Ediciones. 